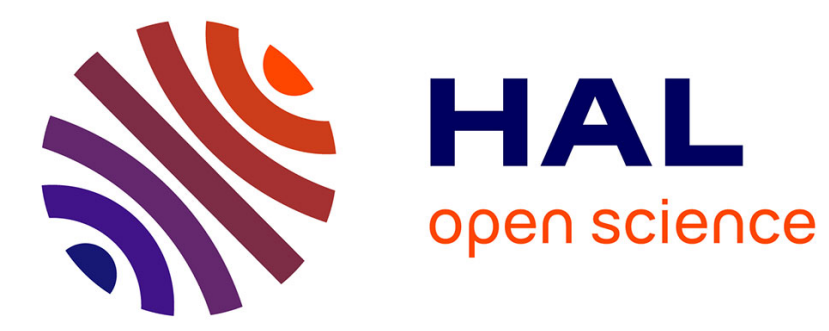

\title{
Tourisme de la nature vivante en Côte d'Ivoire Georges Roure
}

\section{To cite this version:}

Georges Roure. Tourisme de la nature vivante en Côte d'Ivoire. Revue forestière française, 1959, 4, pp.289-309. 10.4267/2042/27495 . hal-03382105

\section{HAL Id: hal-03382105 https://hal.science/hal-03382105}

Submitted on 18 Oct 2021

HAL is a multi-disciplinary open access archive for the deposit and dissemination of scientific research documents, whether they are published or not. The documents may come from teaching and research institutions in France or abroad, or from public or private research centers.
L'archive ouverte pluridisciplinaire HAL, est destinée au dépôt et à la diffusion de documents scientifiques de niveau recherche, publiés ou non, émanant des établissements d'enseignement et de recherche français ou étrangers, des laboratoires publics ou privés. 


\section{TOURISME DE LA NATURE VIVANTE EN COTE D'IVOIRE}

\section{Paysages végétaux, animaux et humains}

\section{I. - Naissance du grand tourisme en Côte d'Ivoire}

\section{Réminiscences et ACtualités}

Ces dix clernières années ont été marquées par un renouveau des aspirations touristiques: alors que le tourisme du demi-siècle écoulé était orienté vers les régions pittoresques et les hauts-lieux d'Europe, le voyageur semble abandonner cette routine pour rechercher l'attrait des terres et cles civilisations africaines.

Ne cherchant plus à se retrouver et à comparer, " il quitte son climat et se dépayse " pour se pénétrer d'images nouvelles et de rythmes différents clont le roman, le reportage et même le cinéma n'ont pu lui rendre la plénitude. avec ses couleurs, ses senteurs et sa vivante atmosphère.

Ce tropisme a d'ailleurs été favorisé par le développement en quantité, en densité et en rapidité, de moyens de transport intercontinentaux et locaux: aux pistes hamacaires ont succédé les routes, wagons-lits et autorails ont remplacé le (' dur ) et les étapes aériennes ont pratiquement supprimé les clélais de transport.

Le prenier rallye international automobile Méditerranée - Le Cap en I950, puis de nombreux voyages touristiques individuels ou collectifs ont démontré complètement que le massif continent africain est maintenant ouvert aux voitures de tourisme. L'affluence des visiteurs par avion, en automobile à travers le Sahara, ou par paquebot, pour fêter la naissance du port d'Abidjan en février I95I, et, plus récemment, le grand rassemblement pour l'inauguration du pont d'Abidjan, confirment cette réalité: la Côte d'Ivoire est bien ouverte au grand tourisme.

Chaque jour, un avion long courrier de grand confort, de l'une des trois grandes compagnies, Air France, U.A.T., T.A.I., assure

(I) Je remercie ici mon ami B. Holas, de l'Institut Français d'Afrique Noire, à Abidjan, qui m'a donné la garantie de son autorité dans les Sciences Humaines, et aux travaux duquel, avec son aimable autorisation, $j$ 'ai fait de larges emprunts (G. R.). 
la liaison Paris-Abidjan, soit par Bordeaux et Bamako, soit par Marseille et Niamey, ou par Dakar, ou par Casablanca. Chaque semaine au moins, un paquebot des Chargeurs réunis de Bordeaux ou de Fabre-Fraissinet de Marseille fait escale à Abidjan.

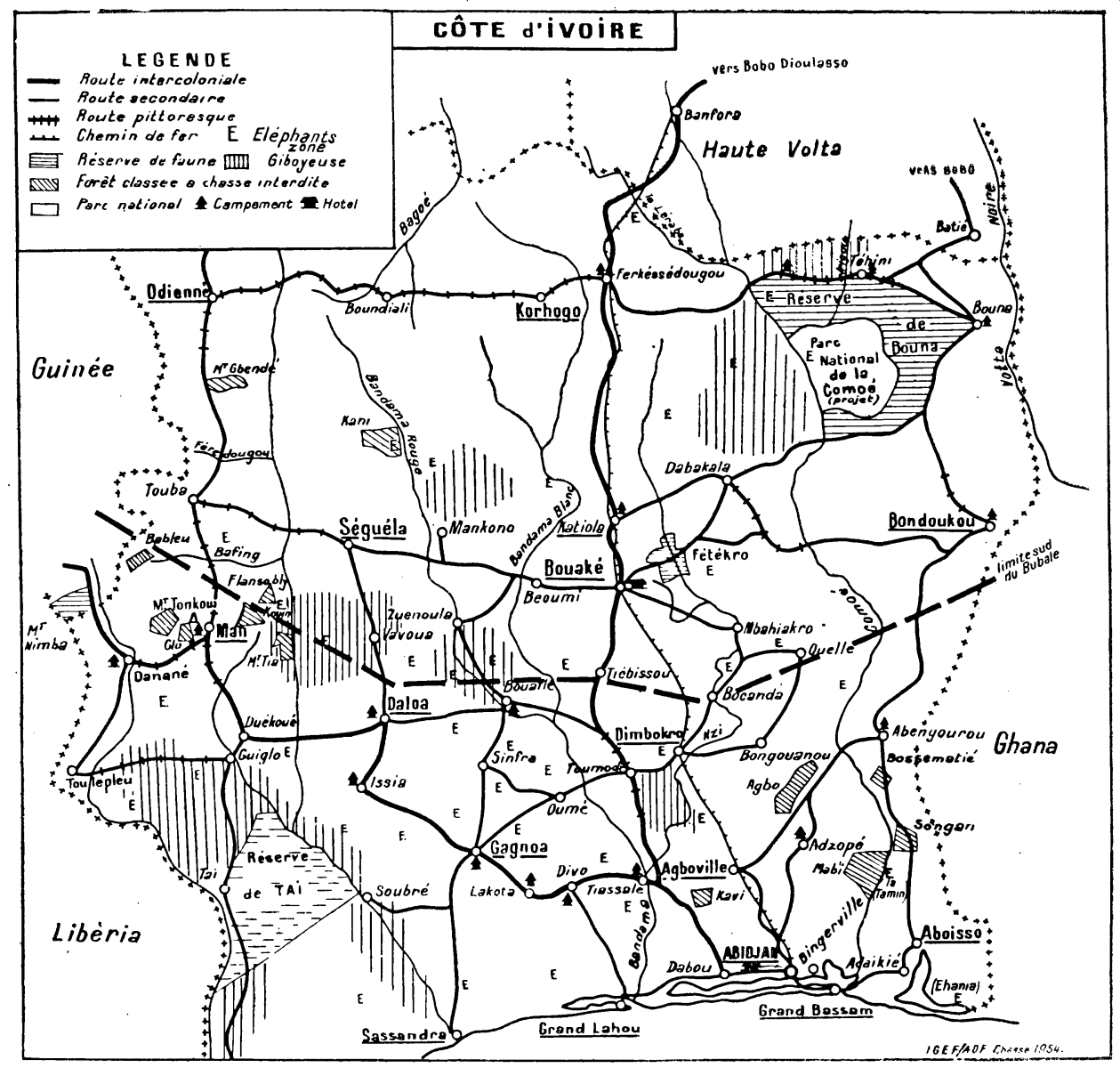

Carte extraite de « Faune et Chasse en A.O.F. 》 par G. Roure.

Le touriste français se présente en Côte d'Ivoire avec sa seule carte d'identité, son inévitable certificat de vaccination antivarioloamarile et son billet de retour ou toute caution équivalente. Si le voyage et les séjours dans les grands centres sont coûteux, il n'en est pas de même en brousse. La bonne époque pour circuler dans le pays sans risques de crues ni tornades, et dans une fraîcheur relative, est celle qui correspond à l'hiver d'Europé, 
Les routes ivoiriennes sont excellentes: 24000 kilomètres de routes et pistes dont Io ooo de routes permanentes et 300 kilomètres bitumés. Des rallyes automobiles ont eu lieu chaque année depuis r955 sur des itinéraires de plus de mille kilomètres, et même un rallye féminin, en 1958 , encore timide il est vrai, puisqu'il ne comptait que onze équipages.

Dans le dépaysement créé par un rapide voyage sous ces horizons nouveaux, il faut au voyageur une réflexion pour comprendre que les valeurs se trouvent inversées. Ce qui est surprenant, ce qui est extraordinaire ici. ce sont les réalisations modernes, les lycées et collèges, les hôpitaux, les mairies, les quartiers modernes, les usines et les entrepôts, les routes et les voies ferrées, les barrages... et toute cette jeunesse, issue de cent groupes ethniques différents, qui va à l'école et parle de son avenir en français.

Dès l'arrivée à Abidjan, et au passage dans les villes principales, ces réalisations, vulgarisées par la presse et la radio, ne peuvent passer inaperçues:

“ La Côte d'Ivoire tourne le dos à son passé ) et se lance hardiment vers l'avenir.

Et pourtant: à part les caravelles lusitaniennes, lancées par l'Infant Louis le Navigateur, qui atteignirent le Cap des Palmes au milieu du $\mathrm{Xv}^{\mathrm{e}}$ siècle pour y troquer étoffes et colifichets contre l'or, à part leurs concurrents dieppois du siècle suivant, puis hollandais au $\mathrm{XVII}^{\mathrm{e}}$ siècle, marins-commerçants, bourlinguant le long de la Côte des Dents - côte cles Malegens à l'Ouest et côte des Bonnes Gens à 1'Fst - vieux vocables de la Côte d'Ivoire, à part nos tentatives de pénétration missionnaire au $\mathrm{xv}^{\mathrm{e}}$ siècle à Assinie, il n'y a guère plus d'un siècle que Bouët-Willaumez signait en I842 les premiers traités avec les chefs côtiers à Assinie, à Grand-Bassam et à Dabou; il n'y a pas trois quarts de siècle que les efforts conjugués des Verdier. Treich-Laplène et Binger aboutissaient aux traités de protectorat sur l'Esr éburnéen, qui mettaient fin à la pression britannique et ouvraient la route par l'Indénié vers Bondoukou, le pays de Kong et le Baoulé, alors qu'à l'Ouest la Convention francolibérienne de I892 fixait la frontière sur le Cavally. La Colonie de 1a Côte d'Ivoire était constituée en I893 sous l'autorité de son plus illustre explorateur, Binger, qui fit progressivement pénétrer et reconnaitre 1'Ouest par les Fleuves Cavally. San Pedro et Sassandra et poussa son influence jusqu'à Man et Touba.

Il avait fallu cinquante ans pour clessiner et reconnaitre la Côte d'Ivoire (I842-I893). " De nombreuses années seront encore nécessaires pour pacifier ce pays cloisonné aux races multiples, farouches, et jalouses de leur isolement " (Ct Chailley) pendant que se met au travail la cohorte des traceurs de routes et des bâtisseurs, des fonctionnaires et des commerçants, que nous jugeons à l'œuvre réa- 
lisée au cours d'un autre demi-siècle, dont le début fut marqué par la construction du wharf de Grand-Bassam (I9OI) et le commencement du chemin de fer (I903) qui allait drainer le Baoulé dès I9r2, l'ouverture de la première école secondaire à Bingerville en r9ro, qui commençait à former les élites, alors que le caféier, essayé dès I89I par. Verdier, était largement introduit en I900 à Bingerville, et que le cacaoyer, apparu en 1895 , prenait de l'extension à partir de rgo8, sources de la richesse économique actuelle.

Une récolte annuelle de $\mathrm{I} 20$ ooo tonnes de café, 80 ooo tonnes de cacao, 35000 tonnes de bananes, du riz, des palmistes, du coton, 250000 tonnes de bois, des diamants, et une industrie déjà prospère de scieries, de conserveries, d'huileries... cela marque l'économie essentiellement paysanne du pays, le plus solide substratum de la nouvelle civilisation montante.

Cet essor économique, qui fait de la Côte d'Ivoire le troisième producteur mondial de café, après le Brésil et la Colombie, et le quatrième pour le cacao après le Ghana, le Brésil et la Nigéria, ne va pas sans modifier profondément l'aspect et la vie du pays, alors que l'effort des bâtisseurs s'épanouit dans les réalisations spectaculaires de ces dernières années, avec l'achèvement de la voie ferrée qui atteint Ouagadougou, l'ouverture du port d'Abidjan dont le trafic dépasse le million de tonnes, la construction des quartiers neufs et des usines de la capitale et la mise en chantier du barrage de la Bia qui fournira l'énergie électrique à toute la région d'Abidjan.

Mais fort heureusement 500000 hectares de café, de cacao et de bananes et un millier de chantiers forestiers, s'ils nécessitent des mesures de sauvegarde pour la conservation de la fertilité et de la productivité du pays, n'ont pas changé la physionomie de la Côte d'Ivoire, et, pour peu que l'on s'écarte des grandes artères routières et des centres de production, on retrouve encore inchangées l'Ame africaine et la nature africaine. Mais, si l'on peut citer ensemble les buildings abidjanais et les soukala lobi, le port d'Abidjan et les pirogues effilées des Krou, les municipalités actuelles et les rites animistes millénaires, les hôpitaux et les pratiques de la sorcellerie, les usines modernes et l'artisanat traditionnel, " c'est parce qu'il s'est opéré une symbiose entre deux genres de vie ».

Que l'on arrive par voie maritime ou par la ligne aérienne de la côte, la zone littorale apparaît comme une interminable plage perpétuellement battue par la barre, uniquement sableuse à l'Est, parsemée de rochers à l'Ouest, seulement coupée par les timides sorties des cours d'eaut. De Fresco jusqu'au Ghana, c'est-à-dire sur la moitié orientale de cette côte un cordon lagunaire de 300 kilomètres de long est en contact immédiat avec la forêt dense.

Derrière cet ourlet littoral, à quelques kilomètres d'Abidjan, 
s'étend la forêt dense qui, vue d'avion, n'est qu'un océan moutonneux, d'un vert sombre, ne laissant rien soupçonner de la structure même de la formation.

Malgré cette apparence massive et simplifiée du premier abord,

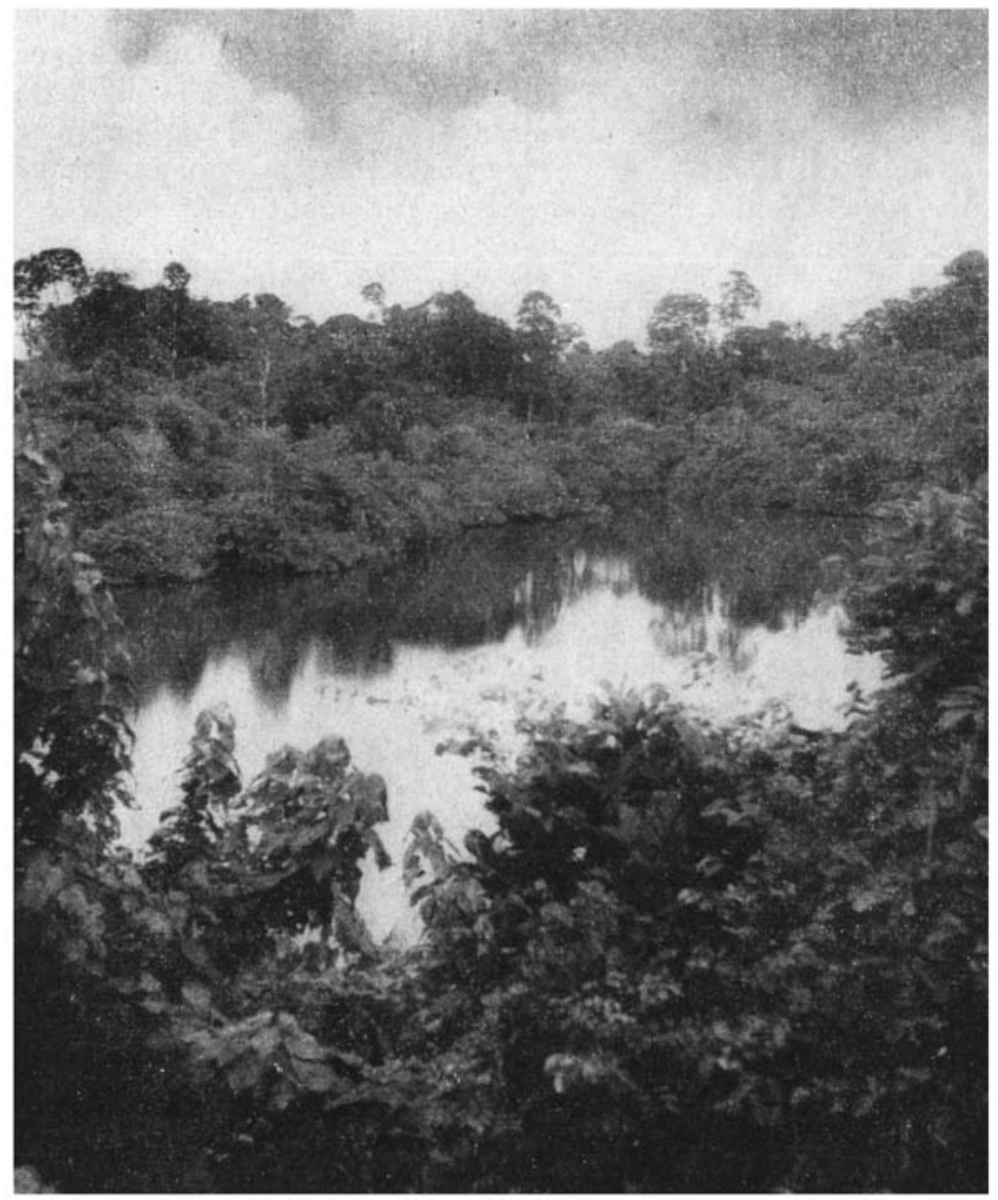

La Basse-Côte et ses charmants paysages lagunaires.

(Cliché Ifan Cocheteux.)

la Côte d'Ivoire est zonée du Nord au Sud et présente une véritable mosaïque ethnique aux cent langages. Elle mérite plusieurs contacts différents. Pour qui dispose d'un bon mois, ou à la rigueur de trois semaines, et d'un bon véhicule automobile, on préconisera un tour de Côte d'Ivoire qui permettra des stations peu nombreuses mais choisies, réunies par des itinéraires typiques. 
A partir d'Abidjan, on peut visiter à petites journées, sans abandonner le confort de la ville, le parc national du Banco, la Basse Côte et ses charmants paysages lagunaires, ses plantations et ses chantiers forestiers; connaître aussi l'aspect humain des faubourgs pleins de vie d'Adjamé et Treichville et y ressentir le formidable appel économique et social de la capitale. On pourra aussi s'imprégner des traditions folkloriques, des coutumes religieuses et des techniques artisanales éburnéennes en visitant le musée d'ethnographie qui abrite les plus importantes collections d'Afrique noire (près de 20000 pièces) et présente des ateliers artisanaux destinés à montrer et maintenir la tradition de la sculpture sur bois, de la poterie et du tissage.

Puis, à travers la forêt, par la route du cacao (Adzopé, Abengourou, Agnihélékrour) et en savane par Bondoukcu (reminiscences de la pénétration et voisinage du Ghana) gagner la Réserve de. Bouna pour observer sa faune protégée en milieu naturel de savane boisée, avec ses ethnies archaiques voisines, I obi et Birifor... et son confortable campement de Ouango-Fitini.

De là, jar la bretelle est-ouest Haute Vclta - Côte d'Ivoire. on lébouche par Ferkéssédougou à Korhogo dans un paysage de savane-parc, déboisée, peuplée et cultivée par la laborieuse et hermétique population Sénoufo, bel exemple de civilisation conservée. arec 111 artisanat remarquable.

T.e paysage de savane, pent-être monotone, mais combien prenant, se poursuit, par Odienné ( « Séguéla, jusqu’à la région de Toula et Man où l'on peut arhirer un autre paysage, montagneux et parsemé cle villages haut perchés, abritant une population attachée encore à certaines vieilles coutumes dont les cérémonies d'excision ef de circoncision sont la plus saisissante expression, avec les grandes fêtes qui accompagnent au début de l'année les sorties solennelles des initiés.

Le long du Cavally, en frontière du. Lilséria, de Toulépleu à Tabou, les Oubi et les Guéré, sociétés naguère isolées, évoluent et s'adaptent aux cultures industrielles, comme leurs voisins Bakoué, Niaboua, Dida et Bété, grands planteurs de café, qui occupent la région forestière voisine, de Daloa, Issia et Gagnoa.

Ia grande artère routière Man, Duékoué, Daloa, Gagnoa, Tiassalé, Abidjan, dessert toute la région forestière du Sud-Ouest de la Côte d'Ivoire, remarquable par ses populations variées, leurs masques et leurs danses, par sa grande faune de " forêt ", par sa vie agricole intense, café et cacao, cultures vivrières, par ses exploitations forestières groupées vers les voies d'évacuation.

Puis, passé Tiassalé et ses plantations de café, cacao, ananas et bananes, passés les beaux sites du Bandama et les magnifiques vestiges de forêt dense, nous trouvons à Ndouci la route bitumée pour traverser les fameuses savanes herbeuses édaphiques, jadis inutiles, 
qui sont maintenant occupées par des plantations industrielles de palmiers à huile, d'hévéas et d'ananas. Puis, c'est, de Dabou à Abidjan, le paysage côtier de brousses inondées et de lagunes, d'où l'on peut atteindre le cordon littoral et ses pittoresques villages de pêcheurs, Songo Agban et Songo Té, sur la lagune Ebrié, Azuréti et Gonzagueville près d'Abidjan.

Après un dernier bain de verdure, à la traversée du Parc National du Banco, on pénètre dans la vie intense des quartiers nord d'Abidjan, bondés de représentants de tous les groupes ethniques venus par cette route de l'Ouest, puis dans le faubourg d'Adjamé proprement dit, demeure des Ebrié autochtones, cultivateurs et pêcheurs, qui ont donné leur nom à la lagune qui fait la richesse et le charme d'Abidjan.

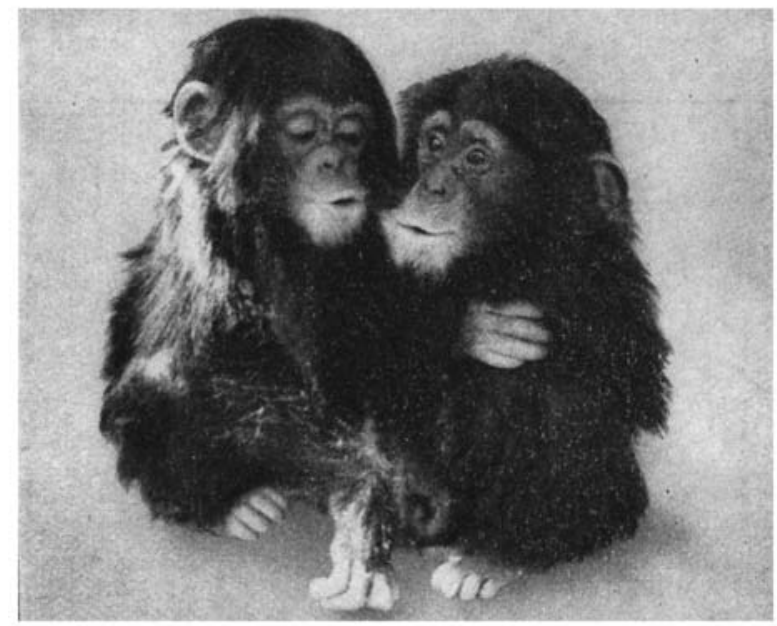

(Cliché Ifan.)

\section{II. - A travers les savanes de la Haute Côte d'Ivoire}

\section{LA RÉSERVE DE FAUNe DE BoUna \\ LES VIEILLES CIVILISATIONS LOBI, SÉNOUFO ET MANDÉ}

Revenons sur les principales étapes de ce circuit touristique de la Côte d'Ivoire.

$\mathrm{Au}$ delà de la bande côtière, la belle route goudronnée, sur laquelle débouchent les chemins desservant chantiers forestiers et exploitations agricoles, traverse la magnifique forêt dense (qui s'étend à l'Est jusqu'au Ghana) coupée de plantations bien ordonnées de caféiers, de cacaoyers et de bananiers, mais aussi de cultures vivrières établies sous le couvert conservé de quelques beaux arbres. 
Chaque village, aux habitations rectangulaires bien alignées, est un centre important de traite du café et du cacao. Malgré cette évolu-

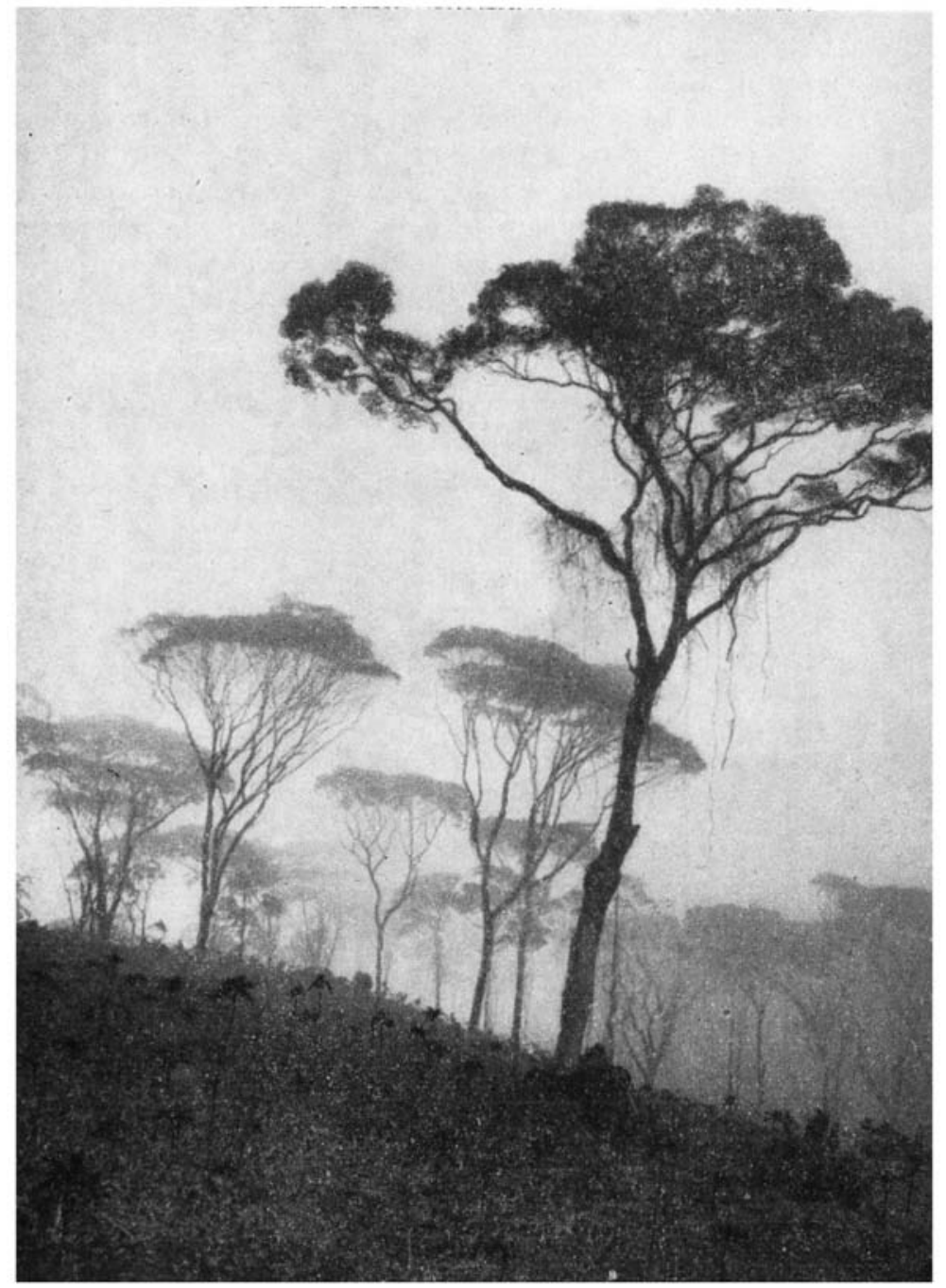

Cultures vivrières sous le couvert de beaux arbres.

(Cliché Pep's.)

tion d'une agriculture presque industrielle, la structure de base des Atié et Agni reste souvent inchangée avec ses particularités culturelles, la technique perfectionnée de ses artisans, dont certains 
sculpteurs ou fondeurs sont d'authentiques artistes; les habitudes vestimentaires traditionnelles se maintiennent et les tissus multicolores, portés drapés " en toge » rehaussent par leur splendeur tous les grands événements rituels dont le plus célèbre est la fête annuelle des ignames, et le plus pittoresque la cérémonie des chaises sacrées à Abengourou.

A mi-chemin entre Abengourou, " capitale du cacao », et Boncloukou, l'oppressante forêt lense, qui noie tout relief, fait place progressivement aux horizons plus dégagés et aux collines pittoresques d'une savane boisée, encore parsemée d'îlots de forêt, de plantations et de cléfrichements sous couvert élevé. Les cases rondes remplacent les construction carrées de la forêt et le vêtement soudanien se mêle à la toge.

A Bondoukou se côtoient les Koulango autochtones, les Dioula envahisseurs commerçants du xve siècle et les conquérants Abron, originaires du Ghana, qui ont conservé l'autorité en la personne du descendant de ce roi des Abron, au village d'Amanvi, qui traita avec Treich-Laplène en 1888 .

La réserve totale de faune de Bouna a été créée en I953 dans le cadre des engagements pris par la France par la Convention de Londres dé I933 relative à la conservation de la faune et de la flore africaines: c'est l' "Unité de protection de la nature » de la Côte d'Ivoire, au mêne titre que le Parc national du NiokaloKobal au Sénégal, la Boucle dı Baoulé au Soudan et le parc du W pour le Niger, la Haute-Volta et le Dahomey.

Dans une pénéplaine granitique portant quelques chaînes et petits massifs montagneux, le paysage dominant est celui de la savane boisée guinéenne, sillonnée d'épaisses galeries forestières le long des rivières, Komoé et ses affluents Bawé, Douin et Iringou, mais coupée aussi de belles futaies de forêt sèche, de plaines d'inondation herbeuses, de plateaux de brousse arbustive et de zones latéritiques plates et nues.

Partout où le couvert forestier ne l'étouffe pas, le puissant tapis de graminées, brîlé en janvier et février par les grands feux de brousse de la courte saison sèche, reprend peu à peu sa croissance quand le paysage reverdit avec les premières pluies de mars-avril; il masque progressivement la faune, pour atteindre 2 mètres et plus de hauteur en pleine saison des pluies.

Cette faune est pour l'essentiel, et ceci est valable depuis Bouna et Bondoukou jusqu'à Odienné et Touba, celle de toutes les savanes boisées de l'Ouest africain, caractérisée, outre le buffle et l'éléphant, par la variété et l'abondance des grandes antilopes, bubale, hippotrague, waterbuck, et des petites et moyennes antilopes de savane, cob de Buffon, redunca, guib, ourébi et céphalophes. Mais ce milieu n'est pas assez soudanais pour qu'on y trouve la 
gazelle à front roux, le damalisque, ni d'ailleurs l'éland de Derby, et il est assez guinéen pour cacher vers le Sud, trop jalousement hélas, quelques belles antilopes de forêt, bongo: et situtonga, et pour montrer des céphalophes de la zone forestière et des singes arboricoles.

C'est dans la partie la plus vive en faune, au Nord, et aux abords de la Komoé, que se trouve le campement de Ouango-Fitini, conçu, construit, organisé et géré par le Service des Eaux et Forêts dans un beau site dégagé de la Réserve, à l'écart de toute localité, répondant ainsi aux impératifs du tourisme de la nature.

Les visiteurs des dimanches et jours fériés ne bénéficient pas pleinement du calme et $\mathrm{d} u$ confort de Ouango-Fitini, mais ceux qui calculent leursi étapes pour venir en semaine sont les bénéficiaires privilégiés d'appartements confortables, d'une salle à manger-bar largement pourvue de réfrigérateurs et de boissons fraîches, et d'un service " à la manière de brousse ».

En fin d'après-midi, ou bien le matin, aux heures où la faune vient s'abreuver aux points d'eau ou en revient, ils sont seuls sur la piste giboyeuse de Bawé et ont ainsi les meilleures chances de surprendre diarra le lion, soli la panthère, un passage de sama l'éléphant, ou le troupeau de sigui le buffle, une harde de sine-sine (cob defassa ou waterbuck), de dagbé (hippotrague ou antilope-cheval), sans parler (les animaux de rencontre presque certaine en toutes circonstances: tangon les bubales, son les cobs de Buffon rassemblés en grands troupeaux près de l'eau, dia ou ngoloni l'ourébi, par couple ou par trio en pleine savane... et les hippopotames et crocodiles, fidèles aux rendez-vous de la mare de Bawé ou du gué de la Komoé au kilomètre 30 de la piste de visite.

On peut encore admirer d'autres petites antilopes, parmi les plus gracieuses, un couple de kongoron (cob des roseaux), ou de mina (guib harnaché) dans une galerie forestière, ou encore quelques-unes des espèces de céphalophes, au nom descriptif de biches-cochons, qui bondissent tête baissée et corps ramassé : le gris (c. de Maxwell), le roux vif à dos noir (c. dorsalis) et le roussâtre aux dos et membres gris (c. rufilatus) et même celui des savanes plus sèches, beige tiqueté de noir (c. de Grimm).

Enfin les troupes agitées de singes: cynocéphales (gbon) en bandes curieuses, singes rouges, cercopithèques hocheur et diane... et, à la tombée de la nuit, des lièvres, et les petits carnassiers en chasse, civette, genette, mangouste, chat sauvage.

Ce n'est pas sans difficulté, on s'en doute, que cette immense réserve, grande comme deux départements français, est protégée des.incorrigibles chasseurs " boucaniers ) des régions voisines dont les plus tenaces sont les Lobi de la Haute Volta.

Pour les touristes chasseurs, qui ne savent se contenter de l'ob- 
servation ou de la photographie, il y a de bons terrains de chasse ouverte au Nord et à l'Ouest de la Réserve.

Quant à ceux qui aiment à faire revivre le passé, ils pourront évoquer dans cette région le passage des anciennes caravanes d'or du Ghana en route vers le monde arabe de Djenné et Tombouctou, ou, sur les maigres placers actuels du pays lobi, la vie des chercheurs d'or venus jadis de Kong et de Bobo-Dioulasso; ou plus proches, les incursions des sofas de Samory, puis les explorations pacifiques des pionniers français aux pays de Kong et de Bondoukou (Binger, Treich-Laplène, Ménard, Marchand, Monteil), enfin les remous de la stabilisation du pays lobi.

C'est au Nord de la Réserve de Bouna que l'amateur d'ethnographie pourra percevoir l'écho des civilisations voltaïques très anciennes, clites paléonigritiques, chez les Lobi qui occupent des maisons fortifiées en terre battue et marchent pratiquement nus; les femmes, avec leurs enfants dans de petits paniers tressés, insèrent dans leurs lèvres des labrets cylindriques de quartz ou de bois dur, et les hommes, chasseurs à l'arc, n'ont pas oublié la fabrication des pointes de flèches. façonnées dans le bois ou le fer, et même taillées dans la pierre. Ils conservent leurs traditions inchangées, funérailles dansées, alimentées par la bière de mil, fêtes d'initiation dioro tous les sept ans, ainsi que leurs intéressants marchés de troc tous les cinq jours.

On trouve une autre civilisation, remarquablement conservée et fidèle aux traditions millénaires, dans la région de Korhogo et Boundiali, formant enclave en pays mandé: les Sénoufo, agriculteurs avisés et laborieux, très attachés à leur terre, ont gardé leurs institutions coutumières, telles que le système initiatique extrêmement compliqué connu sous le nom de poro, qui donne lieu à des festivités extraordinaires, à l'origine hermétiques, mais qui deviennent accessibles à l'étranger.

La coutume millénaire du poro atteint en effet ici son plus haut degré de rigueur, comme chez les Gueraé de la frontière guinéenne, dans des systèmes où le masque figure en tête des agents liturgiques. Des masques de grande taille portés par des forgerons (fono) ou des cordonniers (diéli) figurent des sujets mythiques, généralement zoomorphes. Le sculpteur sénoufo (dalenbélé ou kpembélé) traduit l'idée mythologique par un extraordinaire enchevêtrement d'éléments animaux, gueule de hyène, corne d'hippotrague, crocs de phacochère, avec, au sommet du crâne, un petit calao ou un caméléon qui tient entre ses pattes un récipient destiné à recevoir des substances (" magiques ).

A côté de ces grosses pièces d'un puissant effet dramatique, les kpélé, petits masques commémoratifs, paraissent esthétiquement supérieurs et figurent en place d'honneur chez les collectionneurs. 
Outre les petits objets de fer forgé « travaillés par le forgeron sénoufo et représentant des personnages-génies et des animaux symboles... l'attirail professionnel du géomancien local comporte tout un amas de coquillages cauri, ossements, plumes et peaux de bêtes diverses, galets de quartz, oiseaux, clochettes, anneaux ( magiques ), carapaces de tortues, becs de certains oiseaux, pièces de monnaies anciennes, piquants de porc-épic ) (B. Holas).

De Korhogo à Odiénné, c'est toujours la savane aux boisements discontinus, torturés par l'incendie des hautes herbes qui brîlent

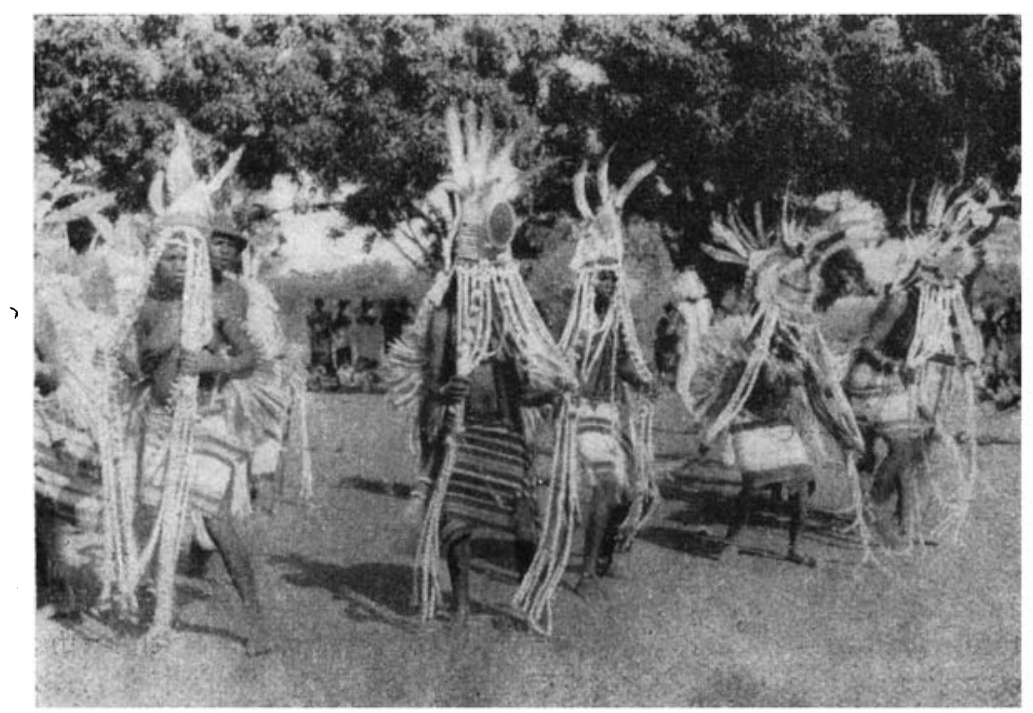

Danse cérémoniale de jeunes filles Sénoufo dans la région de Boundiali.

(Cliché Ifan Labitte.)

en saison sèche, mais cette savane, bien peuplée et déjà proche de la forêt, est assez largement dégagée par les défrichements agricoles qui ne conservent que les arbres utiles: elle prend des aspects de parc ou de " campagne normande ) où les pommiers seraient remplacés par des karité et des néré.

Les Mandé, représentés en premier lieu par les Dioula, qui peuplent cette région, venus jadis du Nord en commerçants pour échanger les tissus contre les colas de la forêt, se sont transformés en bons cultivateurs en dehors de la période du négoce. Le centre historique de leur expansion est l'ancien royaume de Mali qui domina 
tout le Soudan occidental pendant 600 ans, du $\mathrm{XI}^{\theta}$ au $\mathrm{XVII}^{\theta}$ siècle; islamisés dès le XII siècle, les Dioula de Côte d'Ivoire, comme les Malinké-Mandingues du Sénégal et de Guinée, " colporteurs et commerçants, s'infiltrèrent dans les couches aborigènes environnantes pour leur imposer leur hégémonie économique ») (B. Holas).

Dans toute cette région à population assez dense, agricole et travailleuse, il faudra s'éloigner des centres pour atteindre des zones de refuge de plus en plus rares et restreintes, si l'on veut, aidé de quelque vieux chasseur qui vous ressassera les exploits cynégétiques de sa jeunesse, rechercher l'éléphant, le buffle, l'hippopotame, le bubale ou le cob de Buffon, à moins que l'on ne se contente de clébusquer dans les boqueteaux qui bordent les défrichements, les rongeurs ou les oiseaux pillards, lièvre et aulacode, francolin et pintade, ou, par chance, quelque petite antilope rescapée, un phacochère ou une belle outarde.

Toute cette zone des savanes de la Haute Côte d'Ivoire a subi à la fin du siècle dernier les contre-coups de la conquête du Soudan. En effet, alors que la France cherchait à endiguer les empiètements de Samory, dont les Etats formaient écran entre le Soudan et la côte, c'est par Tengréla puis par le pays sénoufo que pénétra Binger: Chargé en i 887 cle relier le Souclan à la côte, il visita à deux reprises Kong, en $\mathrm{r} 888$ et $\mathrm{r} 889$, amorçant des relations commerciales puis réalisant, en $y$ rencontrant Treich-Laplène, la première liaison Soudan - Golfe de Guinée.

Samory, refoulé du Soudan puis de la Guinée, au delà d'Odienné, par les colonnes soudanaises (colonel Combes), mit la main sur la Haute Côte d'Ivoire et chercha à s'y tailler, dans la région de Bondoukou. Kong et Dabakala. un nouvel empire contre lequel échouèrent la colonne Monteil puis les négociations successives. C'est à Man qu'après une lutte de seize ans Samory fut capturé par le capitaine Gouraud.

On lit dans le guide-itinéraire du SITAOF :

Man, 7 ooo habitants, chef-lieu de cercle, PTT, hôpital, missions catholique et protestante. garages, réparations, campement-hôtel de 7 chambres avec restaurant, campement touristique de 8 chambres avec confort à I $\mathrm{km} 500$, courrier automobile, liaison aérienne, aérodrome à $4 \mathrm{~km}$. Centre touristique: cascade, station du Tonkoui ( $340 \mathrm{~m}$ ), plantation de quinquina, massif de Man, ascension de la dent de Man (I IOO m). Tam-tam et danseurs fétichistes $Y a$ couba.

Cette énumération ne rend pas tout le charme et le pittoresque de Man et de ses alentours: site ceinturé de montagnes dentelées portant des cultures de pente et de belles futaies, forêts, clairiérées de puissantes savanes herbeuses, beaux panoramas montagneux et vallées charmantes, populations Yacouba, lointaines alliées des 
Maous de Touba, réfugiées ici depuis des siècles, à la limite de la grande forêt sous la pression de l'invasion mandé.

Cultivateurs de bananes plantain, de riz et de kolatiers, les Dans de Man et Danané, surnommés Yacouba, animistes sous la puissante tutelle du fétiche, extériorisent traditionnellement leur religion par des danses parfois acrobatiques d'équipes spécialisées: danses de garçons au son des tambours, danses d'hommes autour du feu, danses des filles, enfin sorties des masques dont les plus célèbres sont les masques à échasses, sans oublier les exhibitions spectaculaires au cours desquelles de jeunes athlètes font tournoyer par les pieds et se lancent des fillettes danseuses, qu'ils font mine de recevoir sur des poignards dressés à bout de bras.

En frontière du Libéria et de la Guinée se trouve la Réserve naturelle intégrale dı Mont Nimba dont le versant sud est en Côte d'Ivoire et qui constitue un remarquable champ d'étude pour les naturalistes, avec des forêts primaires se prolongeant en galeries atteignant I $\mathbf{5 0 0}$ mètres vers les sommets couverts de prairies d'altitude.

\section{III. - De Man à Abidjan à travers la forêt dense}

\section{E.léphants et masoues, Cafí et bois d'Ceuvre}

A partir de Man nous rentrons, pour un voyage de 600 à I 000 kilomètres suivant les itinéraires, dans cette forêt dense humide que nous avons traversée, un peu rapidement peut-être, d'Abidjan à Abengourou au début de ce circuit touristique.

Nous avons dit que la forêt est le pays de l'éléphant et des masques, du cacao et du bois.

Les masques sont mêlés à toute maniféstation de la vie religieuse, politique et sociale, masques guéré de Guiglo, masques wobé-guéré de la région de Duékoué, masques bétć de la région de Daloa et Gagnoa, masques gouro de Bouaflé; qu'ils soient sculptés dans le bois ou, exceptionnellement, tressés en fibres ou formés de cagoules de tissus, ils étaient à l'origine les porte-parole des dirigeants politiques et religieux. Mais il est presque partout maintenant possible, moyennant une substantielle rétribution, d'organiser une sortie de danseurs masqués ou d'assister à ce spectacle à l'occasion des réjouissances du dimanche ou des fêtes du calendrier officiel.

Dans l'Ouest de la Côte d'Ivoire, le masque en bois est encore l'objet principal du culte des ancêtres et des divinités secondaires. Les masques à visage humain sont extrêmement variés et d'une grande diversité de style: les masques dan (yacouba) représentent un visage idéalisé, front haut, bouche fine, ils recherchent la beauté et la noblesse des traits, fût-ce en les accentuant. L'art guéré-wobé au contraire, oppose ce que P.J.L. Vandenhoute appelle la vision 
réellement ( expressionniste ) au " réalisme idéalisé ) à tendance classique des Dans: il hypertrophie certains éléments principaux du visage, front, yeux, bouche, nez, et en vient même à répéter certains organes jugés plus importants. Une troisième forme de masques combine les éléments du visage humain avec ceux de quelque animal, bec d'oiseau, oreilles de fauve, cornes ou défenses.

I'éléphant est le prince cle la faune forestière, tant pour la population qui craint ses dégâts mais apprécie son énorme masse de viande, que pour les chasseurs à la recherche de son magnifique trophée d'ivoire. De l'éléphant on ne connait généralement en forêt que les larges traces coupant une piste ou longeant un marigot, et seule la chance d'une rencontre ou l'opiniâtreté d'un dur pistage vous met en présence de sa “ masse énorme surgie entre les arbres » la trompe chercheuse tantôt traînant au ras du sol, entre ses deux pointes, tantôt dressée pour interroger le vent, les vastes oreilles battant au rythme de la marche pesante et souple... A moins que la journée de poursuite ne se passe à sentir seulement la présence (" imprécise et intense d'une ombre silencieuse, glissant au travers d'une végétation exubérante sans provoquer autre chose que la chute des gouttes d'eau seconée des feuilles... )

(" Passionnante entre toutes est aussi la chasse au buffle africain, toujours pénible et à l'occasion dangereuse... car l'attaque de près, précédée d'une approche toujours difficile et émouvante, donne à cette chasse son incomparable attrait ")(G. Trial).

L'éléphant de forêt. hien qu'il soit de taille moins élevée que celui de savane, a un aspect plus imposant et plus sombre, un pied plus gros et des pointe's plus fortes et d'un ivoire plus fin, pointes qui, malgré la régression des gros porteurs, donnent encore couramment chez les beaux mâles 50 kilos d'ivoire pour la paire et plus rarement 80 kilos ou plus.

Le buffle nain cle forêt ( $\mathrm{I}$ m au garrot, I7o à 250 kilos) est plus petit que celui de savane avec lequel il est largement métissé vers le Nord, car nombreux sont les animaux de la forêt qui atteignent les savanes préforestières à la faveur des épaisses galeries boisées qui longent les cours d'eau.

La forêt dense est la patrie des singes arboricoles et des chimpanzés, du buffle nain. des belles antilopes bongo et situtonga, de diverses espèces de céphalophes, du grand sanglier noir ou hylochère, l'hippopotame nain et de carnivores typiques poïane, chat doré, sans oublier le léopard. Cette faune de la forêt dense humide, donne l'impression d'être rare car elle est méfiante, cachée et, en grande partie, nocturne.

Colobes agiles aux mains sans pouces, au long pelage varié, toujours perchés dans les frondaisons dont ils se nourrissent: polykomos ou " magistrat " vêtu de noir, à queue blanche, avec une barbe 
et des favoris blancs, colobe bai, noir et roux... Cercopithèques forestiers, à longue queue: mone de pelage gris, membres et queue noirs, hocheur ou " pain à cacheter ), au nez marqué d'une tache blanche, diane à barbiche blanche, mangabey à très longue queue.

Au plus obscur de la forêt se cache le bongo qui rivalise de taille et de cornage avec les belles antilopes de savane, puis le situtonga, au long pelage bariolé de blanc, tous deux si difficiles à apercevoir dans les épais fourrés humides où ils vivent, comme l'hippopotame nain clu Libéria (cantonné entre Cavally et Bandama), amateur de marécages et comme le chevrotain aquatique, petit tragulidé, sorte de " cerviclé sans cornes "), bon nageur, ruminant, mais à loccasion mangeur de poisson.

Gibier plus banal: toute la gamme des céphalophes, petites antilopes aux formes arrondies et près de terre, aux membres fins et courts, caractérisées par une touffe de poils raides entre leurs petites cornes en dards; d'abord deux petites espèces (moins de $40 \mathrm{~cm}$ au garrot) cléjà rencontrées clans les savanes guinéennes, le petit céphalcphe cle Maxwell ou " biche grise ) et le céphalophe à flancs roux ou rufilatus, puis, un peu plus grand, un peu plus sensible à la sécheresse, le dorsalis aux flancs roux vif avec bande dorsale, queue et pattes noires, ensuite les céphalophes strictement forestiers, le noir au pelage brun noir éclairci sur les flancs, le cou et la tête. le zóbré, contonné clans la vallée de Cavally; enfin, à côté de ces petits animaux de dix kilos, se trouvent deux grancles espèces, le céphalophe sylvirultor un céphalophe à dos jaune et le céphalophe de Jentink; le prenier se rencontre anssi dans les galeries forestières de la zone des savanes guninéennes. son pelage sombre est éclairé d'une tache claire en arrière du dos, le second, de tonalité gris clair, est très rare en Côte d'Ivoire, vers le Libéria: tous deux atteignent $80 \mathrm{~cm}$ an garrot et un poids de 70 kilos.

Mais la forêt dense recèle encore bien d'autres petits animaux aux adaptations surprenantes. qui déconcertent le zoolngiste amateur, tel le potamogale. curieux insectivore à l'aspect d'une loutre brune à ventre blanc, adlapté à la vie aquatique; le potto, lémurien proche du singe. minuscule petit " ourson " arboricole de 30 à $40 \mathrm{~cm}$; le pangolin géant et le pangolin à longue queue, rampants st écailleux. qui se nourrissent d'insectes grâce à leur longue langue protractile; le daman (l'arbre, petit animal nocturne, brun et massif, ni ongulé ni rongeur, conramment désigné par son cri alıuha: des rongeurs variés avec l'athérure, maigre cousin du porc-épic, qui ne porte que quelques piquants sur le dos, les extraordinaires anomalures ou écureuils volants qui peuvent faire de grands vols pla-

Danseur.professionnel Yacouba, $\rightarrow$ région de Man.

(Cliché Information Côte d'Ivoire.) 


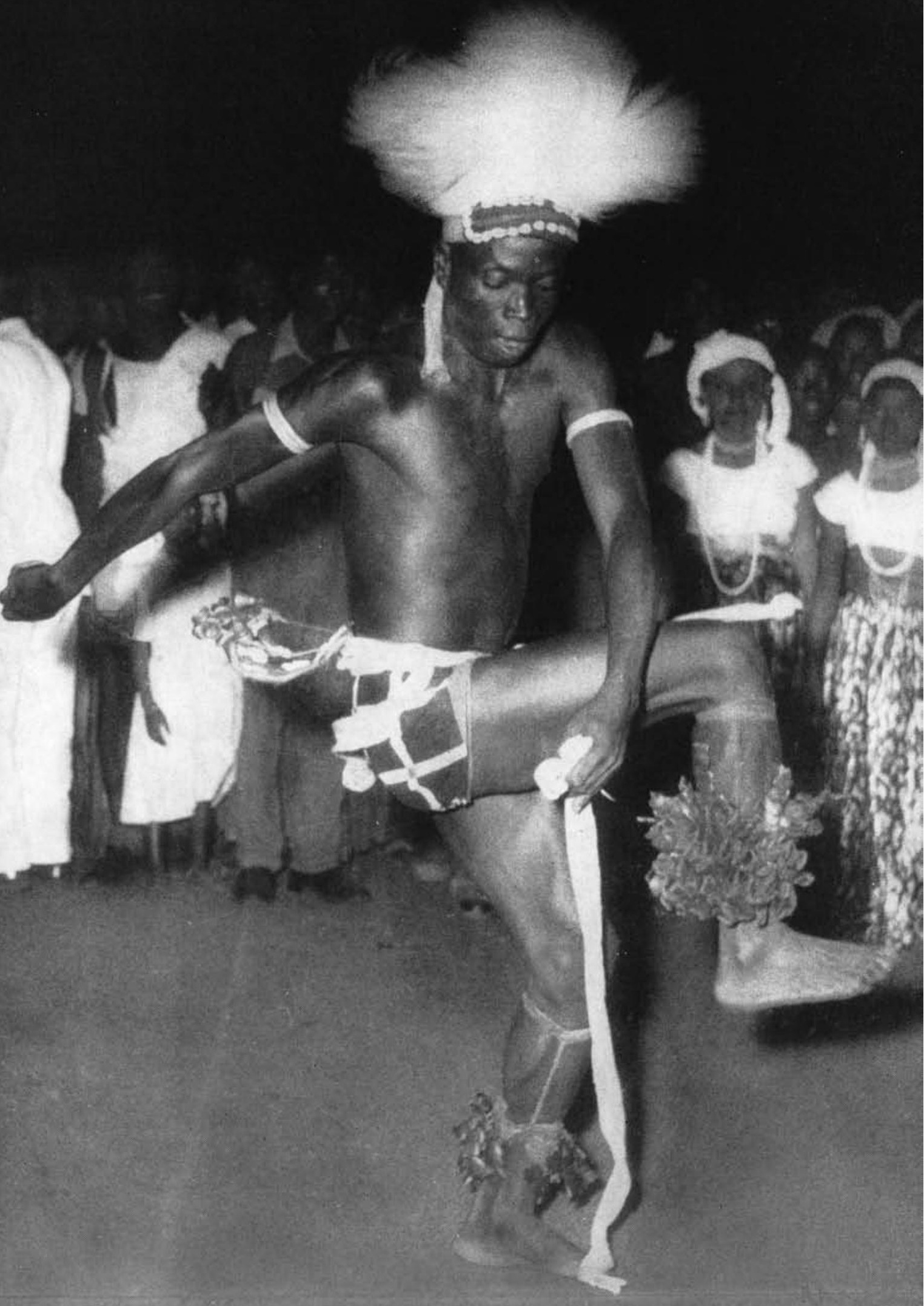



nés grâce à leur membrane parachute tendue entre le membre antérieur et la queue; enfin la charmante et minuscule antilope royale ou néotrague pygmée, qui ne clépasse pas la taille de $25 \mathrm{~cm}$.

Comme carnassiers, outre le léopard et la civette, que l'on rencontre aussi en savane, les prédateurs typiques de la forêt dense sont le chat doré, la genette pardine, la nandinie, la poïane et la petite mangouste brune des marais.

La vue des animaux sauvages sera plus aisée, et leur approche pour la photographie ou le tir sera moins pénible dans la zone des lisières de la forêt dense (Zuénoula, Bouaflé, Toumodi) à la faveur des savanes qui recoupent la forêt et dans lesquelles se rassemblent volontiers les troupeaux. On aura ainsi l'avantage d'apercevoir plus facilement les éléphants et les buffles, mais aussi de trouver encore des antilopes de forêt et des singes arboricoles, et déjà des antilopes de savane, bubale, waterbuck et cob de Buffon.

Nous sommes là en bordure du pays baoulé, qui mérite un détour - et une digression: le pays baoulé, de Bouaké à Dimbokro, apparaît sur la carte Michelin comme un coin blanc de savane rentrant, au centre de la Côte d'Ivoire, dans la masse verte de la forêt. C'est que la mousson océanique du Sud-Ouest, abordant le continent perpendiculairement à la côte libérienne, s'essouffle à la rencontre de l'harmattan sec du Nord-Est, à quelque 350 kilomètres de la côte, sur la ligne Touba, Séguéla, Zuénoula, Bouaflé, Dimbokro. Il en résulte une grande variété des paysages faisant alterner savanes herbeuses semées de palmiers-rôniers et savanes bien boisées, forêts denses peuplées d'irokos et de sambas, ou seulement galeries forestières et îlots de " forêts relictes ), cultures vivrières d'igname, riz, manioc, mais, et aussi plantations de café et cacao au contact de la zone forestière.

C'est là que se sont installés, il y a seulement deux siècles, les Baoulé, venant du royaume de Koumassi (pays achanti), fidèles à leurs cultes et à leurs arts traditionnels. Tisseurs de pagnes et teinturiers, fins sculpteurs sur bois, ils sculptent aussi bien des objets profanes, portes, ustensiles ménagers, sièges, pipes, mortiers, peignes, que des objets rituels, masques, effigies, tabourets et chassemouches de notables. Malheureusement les baoulé laissent tomber en décadence ce travail du bois et ont abandonné la remarquable technique de la fonte à cire perdue, encore pratiquée couramment en Haute-Volta et au Dahomey où elle donne toutes ces charmantes figurines de fonte ou de bronze. Par contre, Katiola a conservé presque intacte une belle industrie très ancienne, strictement féminine, de la poterie, dont on peut faire remonter les techniques inchangées à l'âge de la pierre polie.

$\leftarrow$ En haut: la Dent de Man (Cliché Pep's).

En bas: Train de bois en lagune (Cliché Madec). 
Revenant à notre circuit, c'est dans la zone forestière comprise entre le pays baoulé et la côte que se multiplient les chantiers d'exploitation forestière, principalement le long des voies d'évacuation, routes et chemin de fer, cours d'eau flottables et lagunes navigables; exploitation très dispersée car, de cette forêt dense aux 600 espèces ligneuses, aux 200 espèces de grande taille, on ne tire qu'une douzaine (à la rigueur une vingtaine) de bois commercialisés, et encore à raison seulement d'un arbre par hectare: acajous et similaires, tiama, aboudikro, sipo et makoré, puis avodiré et bossé, pour l'ébénisterie et la menuiserie, iroko, niangon, azobé pour les charpentes, samba et framiré pour le cléroulage et le trancháge.

L'abattage, le tronçonnage et le débardage d'un " géant de la forét ", la mise à l'eau et le flottage des grumes au moment de la crue de l'Agnéby, de la Mé ou de leurs affluents, le remorquage des immenses trains de bois en lagune, autant de spectacles qui méritent l'être vus.

A l'évocation le ce: rivièrés, conme en traversant le Bandama à la sortie de Tiassalé, puis en lébouchant sur la lagune de Grand Lahou ou de I)abou et sur la barre océane, on cloit être renseigné sur les remarquahles possibilités de pêche sportive qu'offre la Côte d'Ivoire.

En rivì̀re on pêche des capitaines d'eau clouce, qui atteignent facilement 5o kilus et se cléfenclent énergiquement, des poissonschiens cu hydrocions de phusieurs kilos, aux mâchoires hérissées de dents, des silures ou gros poissons-chats bruns à moustaches, qui se prennent au fond et y résistent avec force.

lin lagune saumâtre s'ajoutent ethmaloses, mulets, poissons-scies et capitaine: fe me rue guettent les charmants petits villages de pêcheurs dont les pirogues effilées croisent constamment les eaux calmes parsemées d'ingénieux barrages à poissons.

En mer on peut surtout pêcher spertivement les poissons de surface dont certains lépassent couramment un mètre de longueur, capitaine de mer, divers brochets de mer ou barracudas, et le tarpon: de belle taille encore les " rouges ) (lutjanus), les carangues habituées des remous et embouchures, les sérioles, les coryphènes ou daurades, les liches et le tassergal ou faux bar, puis les plus modestes bonites de 2 à 3 kilos.

Requin maquereau, requin marteau, requin tigre, poissons-scie, qui atteignent ou dépassent une longueur de deux mètres et un poids de cent kilos, exigent un équipement spécial. Enfin, des raies de deux mètres et plus peuvent être pêchées au fond ou dans les rouleaux de la barre.

On visitera avec intérêt dans la région de Dabou, le village lacustre sur pilotis de Tiagba et, au bord de la mer, le village aladian de Jacqueville avec ses anciennes maisons européennes, les villages 
adioukrou au moment des fêtes du $l \hat{o} ;$ *lans la région de GrandLahou, les villages des pêcheurs avikam et aladian; aux environs d'Abidjan, les pêcheurs de la plage de Port Bouêt et les pêcheurs de la lagune Ebrié.

Parmi les populations lagunaires ou côtières vivant traditionnellement de la pêche, les Adioukrou, qui occupent la région de Dabou,

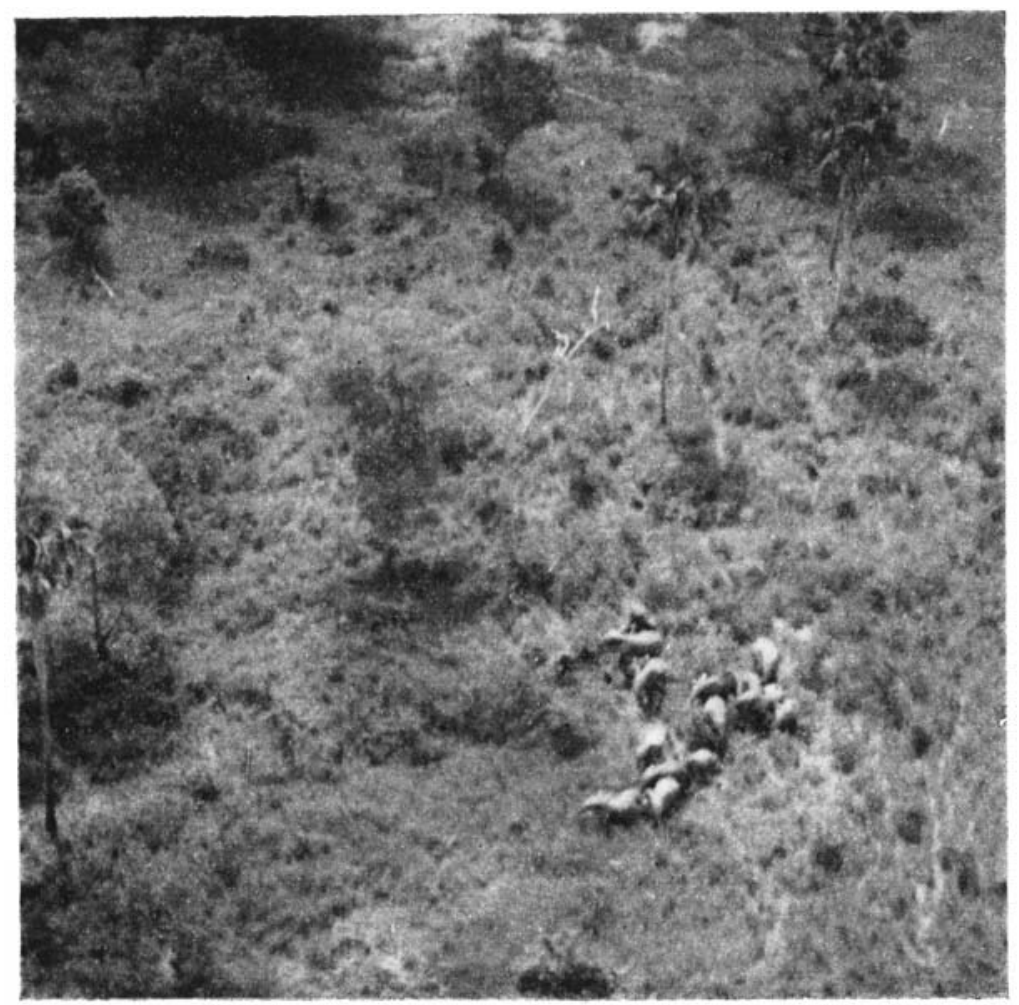

Rassemblement d'éléphants dans la savane.

(Cliché Quijoux.)

ont conservé un système archaïque d'initiation tribale avec hiérarchie sociale basée sur les classes d'âge. Très diffèrents, de souche moins ancienne, mais comme eux bons pêcheurs, sont les Avikam ou Brignan des lagunes de Grand-Lahou à l'Ouest et les Ebrié à l'Est, qui ont des techniques de pêche variées, barrages à poisson, épervier, nasses... mais qui se cantonnent aussi en lagune, au contraire de leurs voisins Aladian ou des Krou de Tabou et des Neyo de Sassandra qui, eux, passent la barre avec leurs longues et robustes pirogues et pêchent en mer au harpon et à la grande senne. 
La plupart des ethnologues sont d'accord sur l'installation relativement récente des populations lagunaires, à l'exception des Adioukrou que l'on rattache, avec les Krou, à des groupements très anciennement présents sous cette latitude. Avec leurs voisins Dida de la Basse-Côte forestière, de type humain bien sylvestre, et surtout les Gagou, de très petite taille, ils constituent, au milieu de la Côte d'Ivoire, sans qu'un lien particulier semble exister entre eux, les noyaux les plus archaiques de peuplement et de coutumes.

Que dire de l'ancienneté de ces civilisations évoquées par la (" magie ) dominant partout les villages que nous traversons?

Bien que l'on ne puisse prouver que l'homme ait participé, au tertiaire, avant l'effondrement de la Mer Rouge, aux grandes migrations afro-asiatiques qui, à travers un Sahara alors florissant, ont façonné les ancêtres de notre faune actuelle, bien que les travaux paléontologiques n'aient pas encore mis à jour dans ces régions des témoignages paléolithiques valables, on aime à imaginer que cette envoûtante forêt fut un refuge mille fois millénaire de l'homme.

A défaut des industries les plus anciennes de la pierre taillée, nous avons des témoignages de civilisation néolithique associant le travail de la pierre polie à l'industrie de la céramique qui s'est perpétuée dans l'artisanat actuel avec ses techniques primitives. Nous avons cles vestiges cle métallurgie autochtone au bord des lagunes, et l'extraction du fer se pratique traditionnellement au NordEst de la Côte d'Ivoire.

En fait, grandes sont les difficulté: pour recréer le passé humain de la Côte d'Ivoire: dans la mémoire du groupe ethnique " le souvenir des premiers occupants de ce pays qui lui appartient en propre aujourd'hui, demeure présent sous la forme d'une tradition folklorique... qui semble accréditer l'existence ancienne de peuplades de petite stature ).

Témoignent en faveur de cette hypothèse: la grancle diffusion du mythe des petits génies et la présence des Gagou, de petite taille, au centre de la Côte d'Ivoire.

( Les récits folkloriques, confondus avec l'imagination populaire vivante, mentionnent des petits êtres rouges, des hommes à queue, des créatures anthropomorphes à pieds retournés... alors que les Guéré, les Wobé, les Oubi connaissent l'existence de collines sacrées, refuges d'êtres humains très méfiants dont seuls quelques vieillards aux yeux exercés aperçoivent parfois les traces ou qu'ils rencontrent sur les sentiers de forêt m.

“ Les Gagou de Oumé donnent une image saisissante de civilisation paléonigritique avec leur agriculture, avec leur cueillette qui garde toujours une place importante, avec leur vêtement en tissu d'écorce battue, avec leur famille patriarcale et leurs clans totémi- 
ques, avec leur animisme où la Terre joue le rôle prépondérant » (B. Holas).

Racines de l'humanité ou implantation des temps historigues?

Forêt-refuge ou d'occupation récente?

Légendes ou réalités?

Pure (" création ") artistique ou production ésotérique?

Techniques archaïques inventées ou introduites?

autant de questions auxquelles la paléontologie, l'ethnographie, la protohistoire apporteront peut-être leurs réponses... à condition que. l'Afrique montante, la nouvelle Afrique, qui clésire, grâce à la mise en commun cles techniques et les ressources de la Communauté, réaliser en cent ans ce que d'autres pays ont fait en mille ans, conserve ses beautés naturẹ̣les et ses sources vives, esthétiques, artistiques, culturelles, et sache découvrir puis sauvegarder les témoignages de ses origines.

Et ceci, qui dépasse largement le cadre des aspirations ou des intérêts d'un renouveau touristique, met en cause la conservation d'une partie du patrimoine du Monde.

Boulaké, novembre 1958.

\author{
Georges Roure, \\ Conservateur des Eaux et Forêts, \\ Chef de la Section \\ Chasse et Protection de la Nature \\ de la Côte d'Ivoire.
}

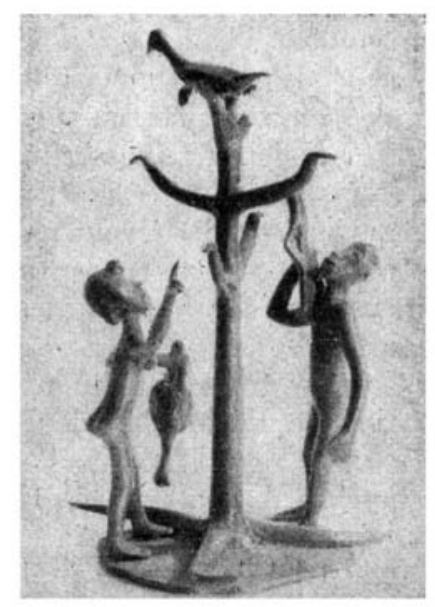

